\title{
Implantable wireless powered light emitting diode (LED) for near-infrared photoimmunotherapy: device development and experimental assessment in vitro and in vivo
}

\author{
Kohei Nakajima ${ }^{1}$, Toshihiro Kimura ${ }^{1,2,4}$, Hideo Takakura $^{1}$, Yasuo Yoshikawa ${ }^{3}$, \\ Atsushi Kameda ${ }^{4}$, Takayuki Shindo ${ }^{4}$, Kazuhide Sato ${ }^{5}$, Hisataka Kobayashi ${ }^{5}$ and \\ Mikako Ogawa ${ }^{1,6}$ \\ ${ }^{1}$ Laboratory of Bioanalysis and Molecular Imaging, Graduate School of Pharmaceutical Sciences, Hokkaido University, \\ Sapporo, Hokkaido, Japan \\ ${ }^{2}$ Savior, Inc., Yokohama, Kanagawa, Japan \\ ${ }^{3}$ Piolax Medical Devices, Inc., Yokohama, Kanagawa, Japan \\ ${ }^{4} \mathrm{~B}$ and Plus K.K., Ogawamachi, Saitama, Japan \\ ${ }^{5}$ Molecular Imaging Program, Center for Cancer Research, National Cancer Institute, NIH, Bethesda, MD, USA \\ ${ }^{6}$ Presto, Japan Science and Technology Agency, Kawaguchi, Saitama, Japan \\ Correspondence to: Mikako Ogawa, email: mogawa@pharm.hokudai.ac.jp \\ Hisataka Kobayashi, email: kobayash@mail.nih.gov \\ Keywords: light emitting diode (LED); wireless power transfer; phototherapy; near-infrared photoimmunotherapy (NIR-PIT) \\ Received: February 16, $2018 \quad$ Accepted: March 21, $2018 \quad$ Published: April 13, 2018 \\ Copyright: Nakajima et al. This is an open-access article distributed under the terms of the Creative Commons Attribution License \\ 3.0 (CC BY 3.0), which permits unrestricted use, distribution, and reproduction in any medium, provided the original author and \\ source are credited.
}

\section{ABSTRACT}

Purpose: The aim of this study was to develop and assess a novel implantable, wireless-powered, light-emitting diode (LED) for near-infrared photoimmunotherapy (NIR-PIT). NIR-PIT is a recently developed cancer therapy that uses NIR light and antibody-photosensitizer conjugates and is able to induce cancer-specific cell death. Due to limited light penetration depth it is currently unable to treat tumors in deep tissues. Use of implanted LED might potentially overcome this limitation.

Results: The wireless LED system was able to emit NIR light up to a distance of $20 \mathrm{~cm}$ from the transmitter coil by using low magnetic fields as compliant with limits for use in humans. Results indicated that the LED system was able to kill tumor cells in vitro and to suppress tumor growth in implanted tumor-bearing mice.

Conclusions: Results indicated that the proposed implantable wireless LED system was able to suppress tumor growth in vivo. These results are encouraging as wireless LED systems such as the one here developed might be a possible solution to treat tumors in deep regions in humans. Further research in this area would be important.

Materials and Methods: An implantable LED system was developed. It consisted of a LED capsule including two LED sources and a receiver coil coupled with an external coil and power source. Wireless power transmission was guaranteed by using electromagnetic induction. The system was tested in vitro by using EGFR-expressing cells and HER2-expressing cells. The system was also tested in vivo in tumor-bearing mice. 


\section{INTRODUCTION}

Near-infrared photoimmunotherapy (NIR-PIT) is a new type of cancer photo-therapy based on NIR light and antibody-photosensitizer conjugates [1]. When the conjugates bind to target cells' membranes and are exposed to NIR light, cancer specific cell death is induced without side effects, leaving adjacent normal cells undamaged [2]. A clinical trial on patients with inoperable head and neck cancer, who cannot be satisfactorily treated with chemotherapy or radiation therapy was approved by U.S. Food and Drug Administration in 2015 (https:// clinicaltrials.gov/ct2/show/NCT02422979). The trial went through phase I/II successfully, suggesting that NIR-PIT may be a promising cancer treatment.

However, the procedure is not exempt from limitations. It is difficult to treat deep regions because of light absorption by tissues [3,4]. In principle, to treat deep lesions NIR light could be delivered by using thin fiber diffusers almost anywhere in the body, e.g. via endoscopes, catheters, or needles $[5,6]$. However, this procedure is a little invasive, especially considering the need for repeated treatments. In fact, repeated treatments or even repeated light exposure on a single dosing of antibody-photoabsorber conjugates can yield better therapeutic effects than a single treatment or a single light exposure $[7,8]$. To try to limit the invasive character of repeated treatments in deep tissues, at the same time preserving efficacy, we investigated the possibility of using implanted NIR light emitting diode (LED) sources. Implanted LEDs are promising as they can be stable light sources for internal or interstitial NIR light exposure, thus enabling repeated light exposure of cancer lesions in deep tissues with minimally invasive procedures.

In this study, we developed a small implantable wireless LED for NIR light exposure and we have tested it in vitro as well as in vivo in tumor bearing mice. To avoid potentially harmful implantation of batteries, power supply to the implanted LED was guaranteed by electromagnetic induction from an external transmitter coil to an implanted received coil coupled with the LED. The electromagnetic field was set to be compliant with standard limits for use in humans, i.e. lower than $0.1 \mathrm{mT}$.

\section{RESULTS}

\section{Wireless NIR-LED}

As shown in Figure 1, we have developed the wireless NIR-LED system by coupling an external transmitter coil with the LED capsule, which included two LED sources and a receiver coil. Energy loss between the
A

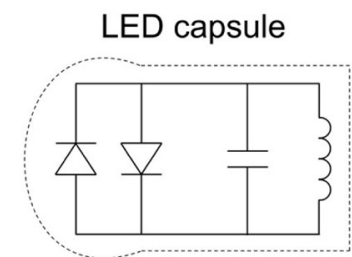

Wireless power supply device

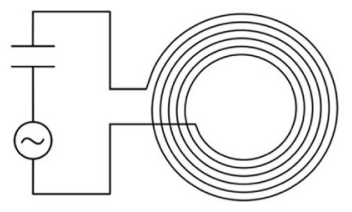

B

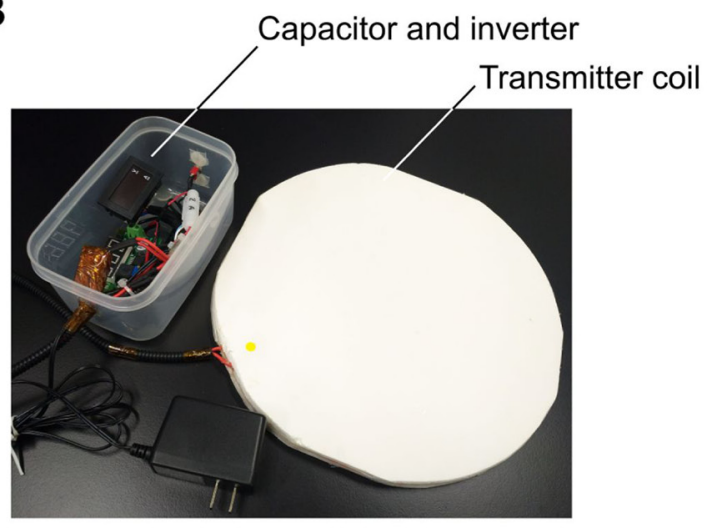

C

D

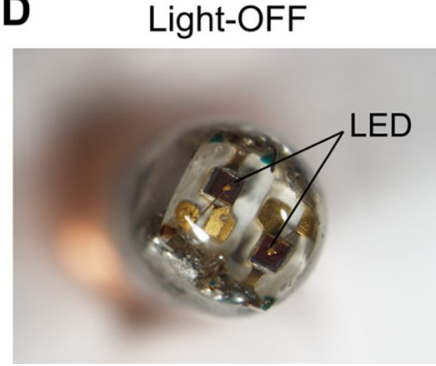

Light-ON

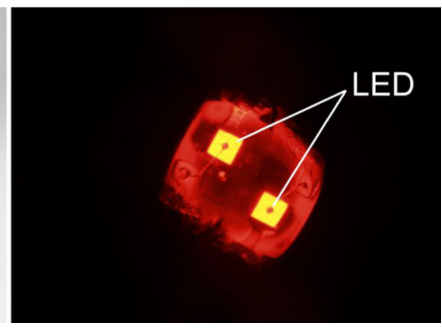

Figure 1: Implantable NIR-LED system. (A) Circuit diagram of the wireless power supply device and the LED capsule. (B) Photograph of the wireless power supply device. The inner and outer diameters of the transmitter coil were $150 \mathrm{~mm}$ and $300 \mathrm{~mm}$, respectively. (C) LED capsule. The length is about $7 \mathrm{~mm}$. A copper wire was winded around a cylindrical ferrite by approximately 30 turns. The LED capsule was coated with biocompatible transparent epoxy resin. Scale bar: $5 \mathrm{~mm}$. (D) Two LEDs at the head of the capsule in light-ON (680-700 $\mathrm{nm})$ and light-OFF state. 
coils was minimized by using a cupper litz wire to limit the skin effect $[9,10]$. Inductance and quality factor were maximized by using high magnetic permeability ferrite in the receiver coil [10]. Power transmission was further improved by limiting the error value of the resonant frequency below $0.5 \%$. The transmitter coil was able to provide power to a receiver at a distance of up to $20 \mathrm{~cm}$ in low power $(\sim 7 \mathrm{~V}, \sim 0.6 \mathrm{~A})$.

\section{In vitro NIR-PIT for EGFR-expressing cells}

Figure 2A shows the effect of NIR-LED irradiation on EGFR-expressing cells in vitro. The cytotoxic effect of NIR-PIT with the wireless LED was evaluated by using EGFR-expressing A431-GFP-luc cells. Severe cell damage such as cell swelling and bleb formation were induced by the combination of Pan-IR700 incubation and NIR light exposure (Figure 2A). Dead cell staining by using EthD1 demonstrated that the uptake was increased in a light dose-dependent manner. No changes were observed with Pan-IR700 alone or with NIR light irradiation alone.

Decreased bioluminescence from NIR-PIT-treated cells indicated early therapeutic effects of NIR-PIT in vitro [11]. As shown in Figure $2 \mathrm{~B}$, bioluminescence imaging (BLI) demonstrated decreased luciferase activity in a light dose-dependent manner. The area with decreased luciferase activity increased with increasing light dose. These results suggested that the wireless LED induced NIR-PIT on A431-GFP-luc cells. The observed characteristics of cell death were the same as those reported in previous studies [1, 12].

\section{In vitro NIR-PIT for HER2-expressing cells}

Figure 3 shows the effect of NIR-LED irradiation on HER2-expressing cells in vitro. Cell swelling and bleb formation were observed in NIR-PIT $(+)$ cells, and the severity of cell damage was dependent on the light, i.e. increased damage was observed with increasing lightdose. Fluorescence imaging showed that EthD-1 uptake increased with increasing light-dose, and that there was no cytotoxicity associated with Tra-IR700 alone nor with exposure to NIR light.

\section{In vivo NIR-PIT}

To assess the effects of NIR-LED exposure in vivo, we conducted experiments on tumor-bearing mice injected with A431-GFP-luc cells on both sides of the dorsum, as shown in Figure 4. Figure 5 shows the results observed in treated and non-treated mice. On day 1, PanIR700 accumulated in both tumors (Figure 5A). On day 2, fluorescence signals of IR700 on LED $(+)$ _NIR $(+)$ tumor was significantly decreased in NIR-PIT-treated mice compared to non-treated (Figure 5A, 5B). On the other hand, there was no difference in relative signal intensity between LED $(+)$ _NIR $(-)$ tumor and LED (-)_NIR $(-)$ tumor in non-treated mice. These results suggested that LED $(+)$ NIR $(+)$ tumors were exposed to NIR light effectively [12]. On day 6, GFP fluorescence imaging demonstrated decreased signals of GFP from LED $(+)$ NIR (+) tumor (Figure 5C), suggesting therapeutic effects of NIR-PIT $[13,14]$. The LED $(+)$ NIR $(+)$ tumors in treated mice were smaller in size than LED $(-)$ NIR (-) tumors (Figure 5D). Moreover, tumor growth was significantly suppressed in LED $(+)$ NIR $(+)$ tumors compared to LED $(-)$ NIR $(-)$ tumors in treated mice (day 4, 5, and 6: $p=0.066,0.011,0.010$, respectively) (Figure 5E). No significant difference was observed between treated and non-treated mice in LED (-)_NIR (-) tumors (Figure 5F). The body weights of the mice were not changed during the experiments, indicating minimal systemic toxicity due to NIR-PIT and the implanted wireless LEDs (Figure 5G).

Overall, these results suggested that the developed wireless LED was able to provide NIR-PIT in vivo and reduce tumors in mice.

\section{Histological analysis}

Figure 6 shows histological analysis in treated and non-treated mice. H\&E staining of LED $(+)$ _NIR $(+)$ tumors in treated mice demonstrated significant cell death (Figure 6A) whereas no apparent damage was observed in the other tumors, neither LED (+)_NIR (-) tumors in nontreated mice nor in LED (-)_NIR $(-)$ tumors in treated and non-treated mice (Figure 6B).

\section{DISCUSSION}

In this study, we have developed a prototype of implantable, wireless powered LED system for NIRPIT. We used wireless power transmission through electromagnetic induction for supplying power from outside of the body to a receiver coil in the LED capsule, so to avoid implantation of batteries. Although the higher the electromagnetic field, the stronger the LED emission, the electromagnetic field in our system was limited to comply with the International Commission on NonIonizing Radiation Protection (ICNIRP) guideline [15] so to avoid possible adverse effects on the human body [16]. To limit possible immune response, the LED capsules were fully coated with biocompatible epoxy resin [17]. Interestingly, our wireless LED system could emit NIR light up to a distance of $20 \mathrm{~cm}$ from the power supply. This means that, ideally, most lesions in the human body can be potentially reached by irradiating from around the body. Wireless powered LED systems such as the one here proposed might potentially become devices for use in humans. Future studies on safety, efficacy, compatibility, and optimal clinical use are necessary.

Another important aspect for optimal development of implanted NIR-LED systems is related to reaching 
a trade-off between the light dose, which is desired to be high for increased treatment benefit, and the electromagnetic field, which needs to be low to avoid harmful effects on the human body. As a result, LED emission is limited due to limited electromagnetic field. Moreover, LED emission is also dependent on the distance between the LED/receiver coil and external transmitter coil and, also, between the small size of the LED capsule and receiver coil. Thus, especially in view of future applications in humans and in clinical settings, maximization of the LED emission is a key design issue. To try to partially tackle this issue, we reduced the error of resonant frequency between the transmitter and receiver coils and we improved the quality factor by using ferrite which has high magnetic permeability. The resulting LED emission was sufficient for treating tumors in mice.

The cytotoxic effects of NIR-PIT does not relate only to the power density $\left(\mathrm{mW} / \mathrm{cm}^{2}\right)$ of the light source, but rather depends on the light dose in the tissue $\left(\mathrm{J} / \mathrm{cm}^{2}\right)$ $[11,18]$. It was reported that light doses in the range 50$150 \mathrm{~J} / \mathrm{cm}^{2}$ can be effective for NIR-PIT $[11,19,20]$. In our in vivo experiments we had a maximum light dose of $320 \mathrm{~J} / \mathrm{cm}^{2}$ which is above the minimal range and, as such, enabled tumor treatment. Another advantage of the proposed LED system compared to other solutions for NIR-PIT in deep tissues is related to the fact that the LED system is small and, once implanted, can irradiate tumors over a long period. On the other hand, conventional implantable light-delivering devices such as catheters

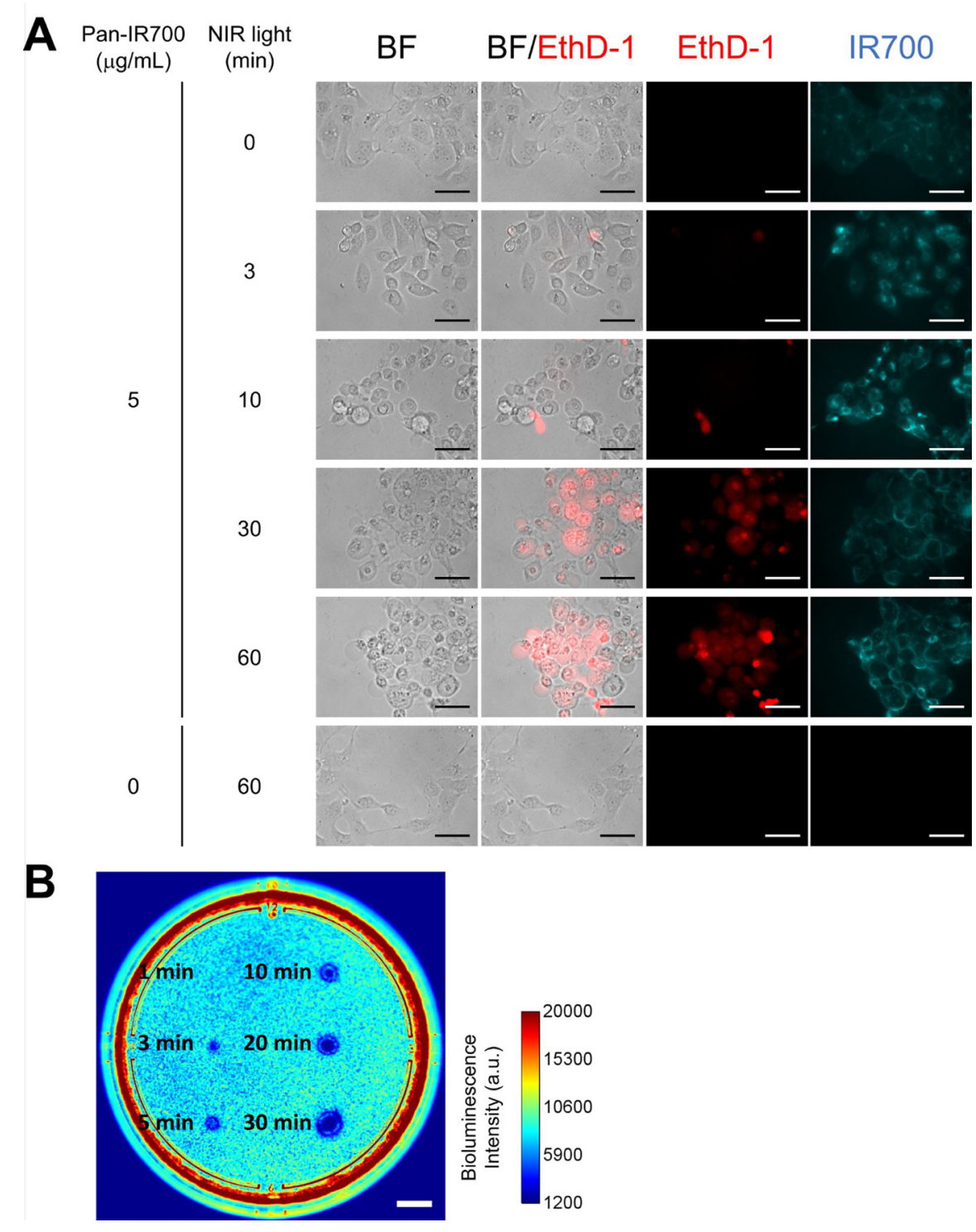

Figure 2: In vitro NIR-PIT for A431-GFP-luc cells. (A) Phase and fluorescent microscopy of NIR-PIT treated A431-GFP-luc cells, which were pre-incubated with Pan-IR700 $(5 \mu \mathrm{g} / \mathrm{mL})$ at $37^{\circ} \mathrm{C}$ for $1 \mathrm{~h}$. NIR-PIT induced cell death with cell swelling and bleb formation. EthD-1 staining showed cell death in a light dose-dependent manner. Scale bar: $50 \mu \mathrm{m}$ (original magnification, $40 \times$ ). (B) Bioluminescence imaging of a $10 \mathrm{~cm}$ dish demonstrated that luciferase activity in A431-GFP-luc cells decreased with increasing light dose. Scale bar: $1 \mathrm{~cm}$. 


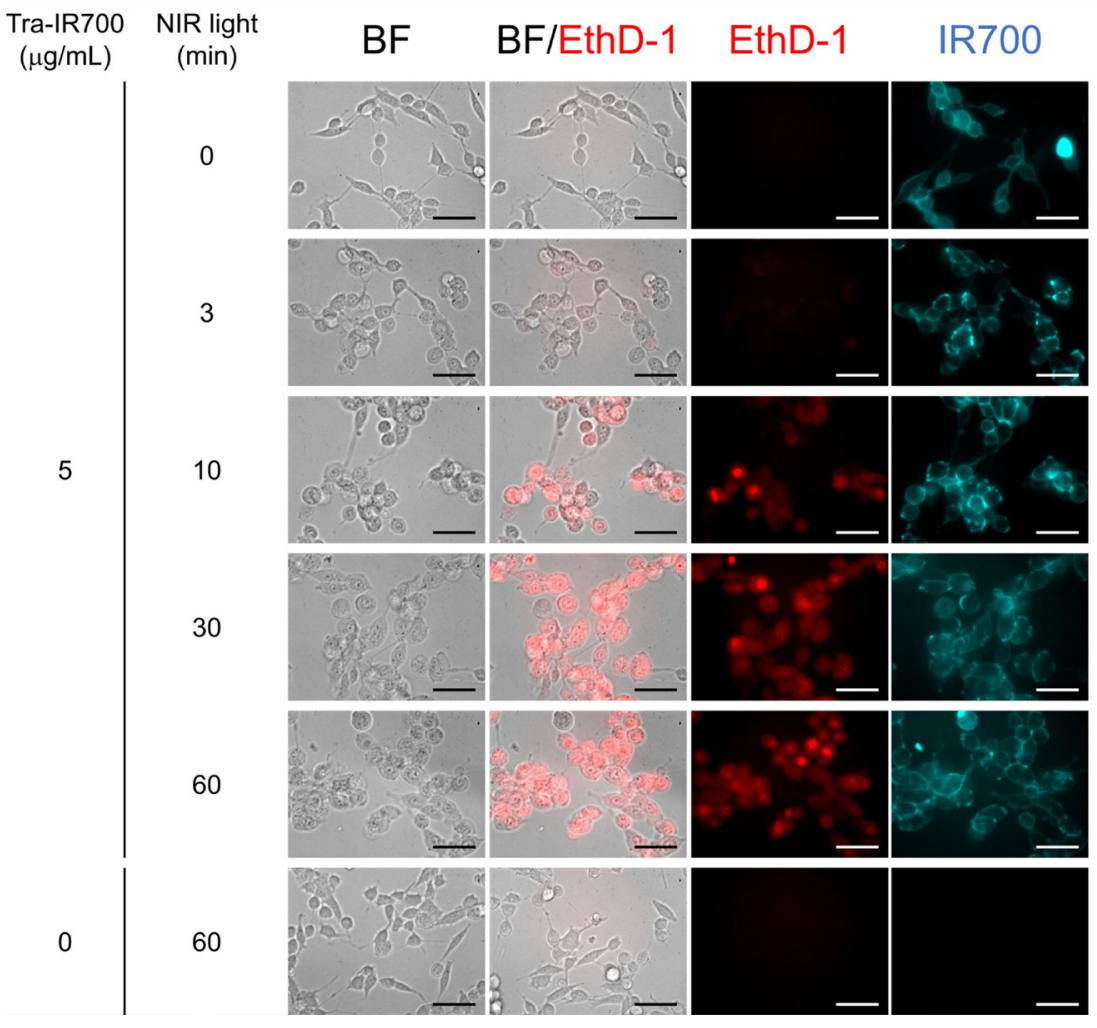

Figure 3: In vitro NIR-PIT for 3T3-HER2 cells. Phase and fluorescent microscopy of NIR-PIT treated 3T3-HER2 cells, which were pre-incubated with Tra-IR700 at $37^{\circ} \mathrm{C}$ for $1 \mathrm{~h}$. NIR-PIT induced cell swelling and bleb formation. The severity of cell damage and the uptake of EthD-1 increased with increasing light dose. Scale bar: $50 \mu \mathrm{m}$ (original magnification, $40 \times$ ).
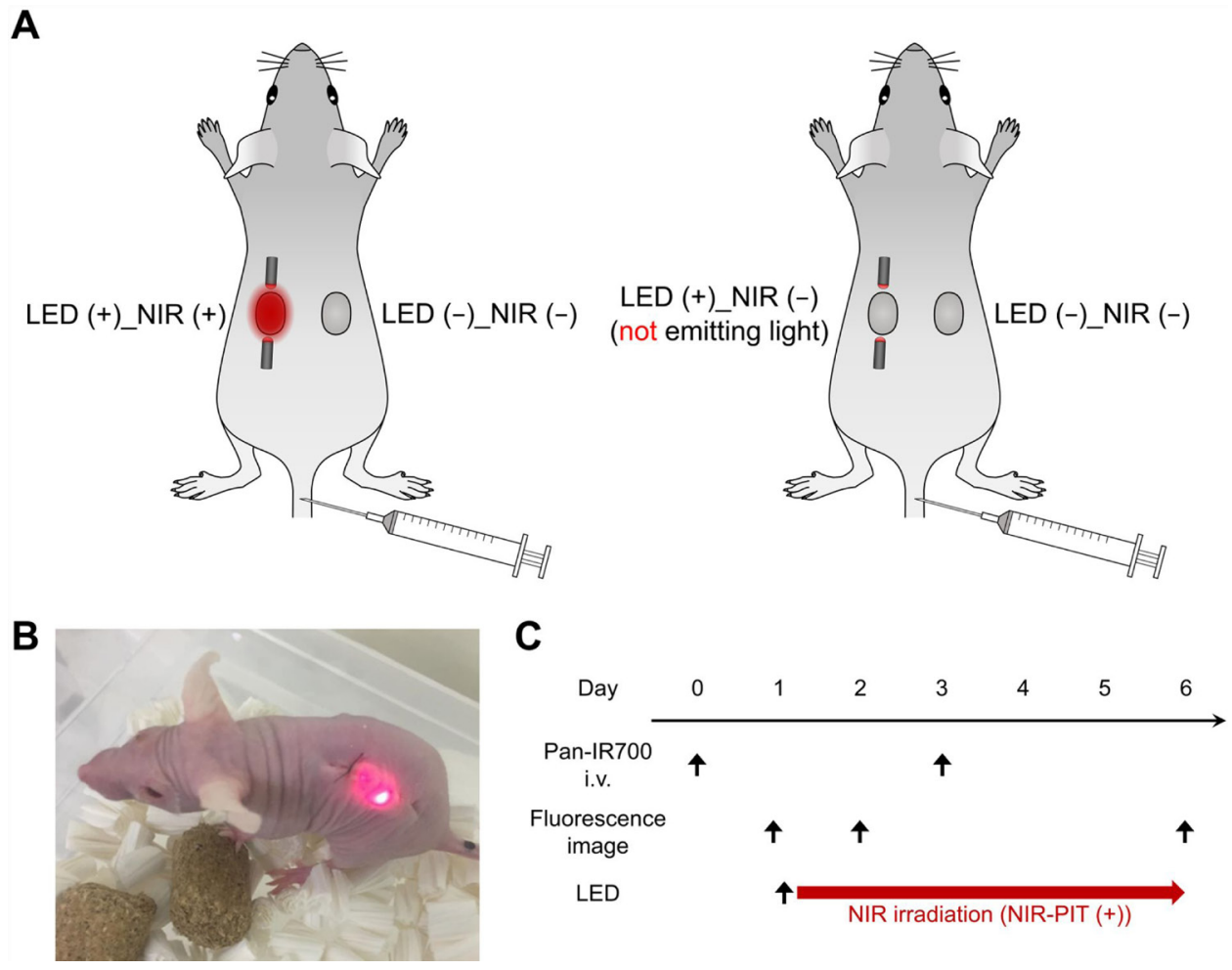

Figure 4: In vivo NIR-PIT in mice: setup. (A) Schematic of tumor-bearing LEDs implanted mice. Treated mice (left): NIR-LED $(\operatorname{LED}(+)$ NIR $(+))$ was implanted in one tumor and no LED in the other tumor (LED $(-)$ _NIR $(-))$. Non-treated mice (right): dummy LED (LED (+)_NIR (-)) was implanted in a tumor and no LED in the other tumor (LED (-)_NIR (-)). (B) Photograph of a treated mouse implanted with the wireless LED. (C) Treatment and imaging schedule. 


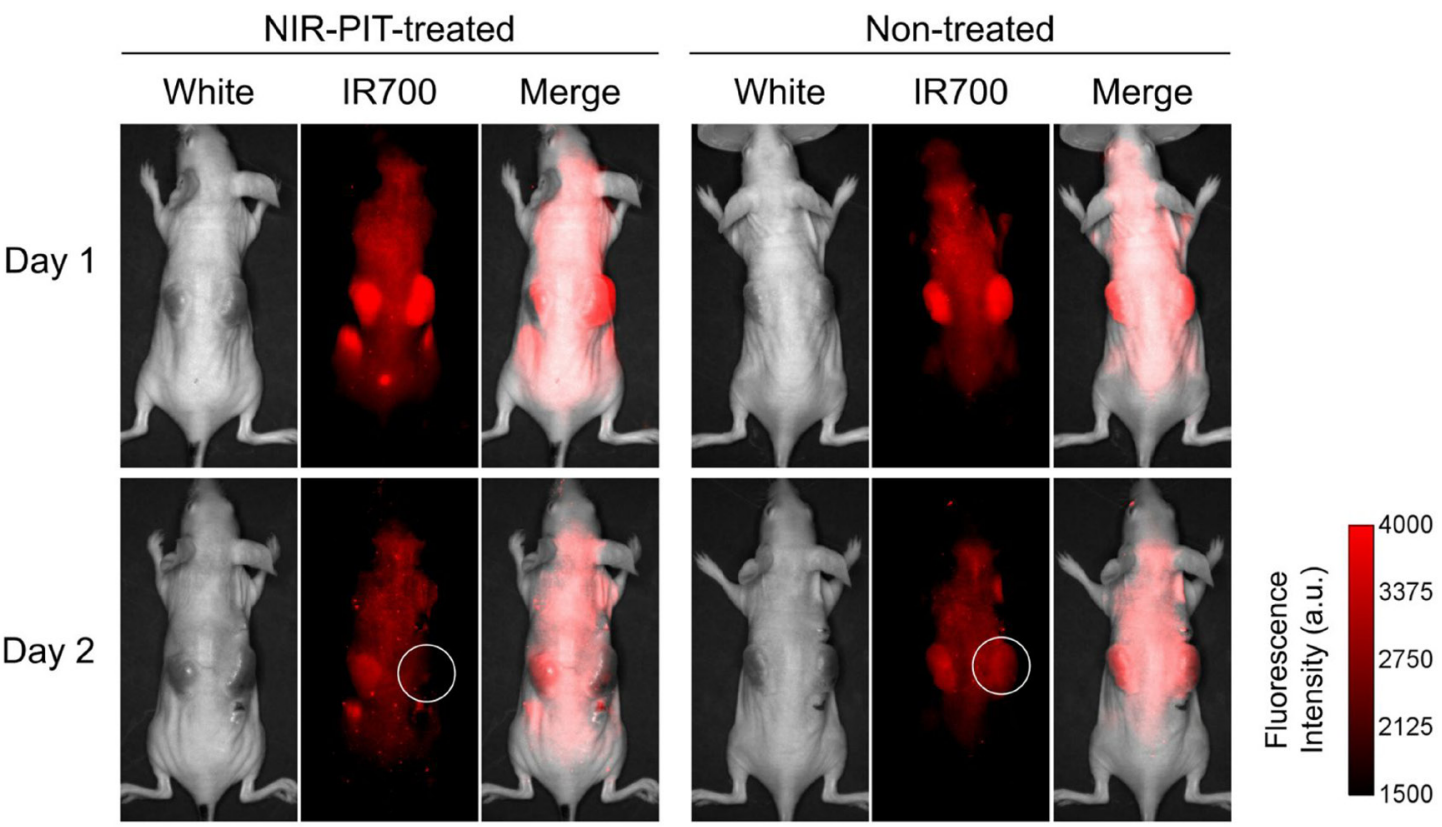

B

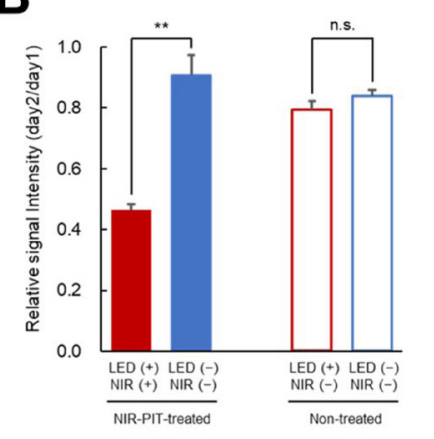

E

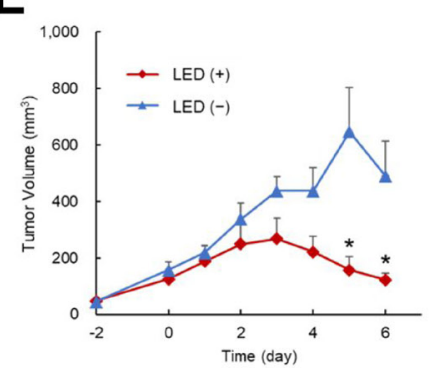

C NIR-PIT -treated

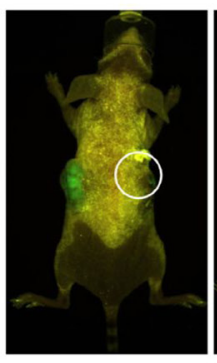

F

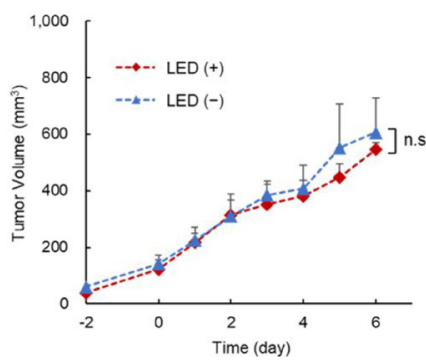

D

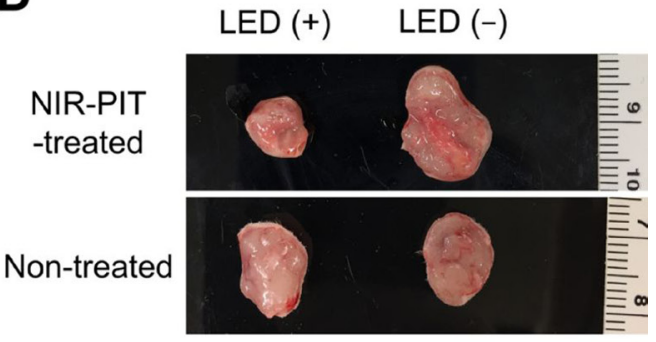

G

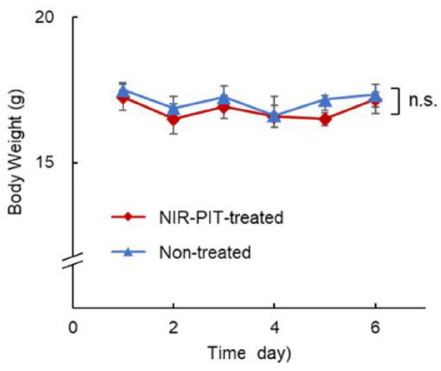

Figure 5: In vivo NIR-PIT in mice: results. (A) IR700 fluorescence images on day 1 (top) and day 2 (bottom) of treated (left) and non-treated (right) mice. White circles indicate the NIR-LED in treated mice and the dummy LED in non-treated mice. (B) The relative signal intensity (day2/day1) of IR700 on LED $(+)$ _NIR $(+)$ tumor in treated mice was significantly lower than in tumors with no LED whereas no significant difference in relative signal intensity between the dummy LED (LED (+)_NIR $(-)$ ) tumor and the tumor with no LED was detected in non-treated mice $\left(n=6\right.$ treated mice, $n=3$ non-treated mice) $\left.{ }^{* *} p<0.01\right)$. (C) GFP fluorescence imaging on day 6 showed that the intensity in LED $(+)$ NIR $(+)$ tumors in treated mice was lower than in (LED (+)_NIR $(-))$ tumors in non-treated mice. (D) Ex vivo images of tumors in treated (top) and non-treated (bottom) mice, showing reduced tumor size in NIR-LED exposed tumors compared to dummy LED and to tumors with no LED. (E) Tumor volume as a function of time in LED (+) NIR (+) tumors and LED $(-)$ NIR $(-)$ tumors in treated mice. The chart shows that tumor growth was suppressed in LED $(+) \_$NIR $(+)$tumors $(n=6$ treated mice). The differences between LED $(+)$ _NIR $(+)$ and LED $(-)$ NIR $(-)$ tumors were statistically significant on day 5 and $6\left(^{*} p=0.011, p=0.010\right.$, respectively). (F) Tumor volume as a function of time in LED $(+)$ _NIR $(-)$ tumors and LED $(-)$ _NIR $(-)$ tumors in non-treated mice. No significant difference in volume was observed between the two tumors in non-treated mice $(n=3$ non-treated mice). (G) Body weight as a function of time in treated and non-treated mice. The chart shows that body weights did not change during experiments in neither group ( $n=6$ treated mice, $n=3$ non-treated mice). 
cannot be left in the body for a long period and require recurrent implantation.

It was proven that the therapeutic benefits of NIRPIT can be enhanced by repeated drug administration and light exposure [7]. The circulating mAb-IR700 penetrates tumors more deeply following the first NIR light exposure, thus enabling more effective treatment $[21,22]$. In our in vivo experiments tumors were exposed to NIR light continuously following implantation. During the treatment period, it is likely that the circulating mAb-IR700 from the first and subsequent injections accumulated in the tumor and contributed to more effective treatment. Use of NIR-PIT with wireless powered implanted LED might potentially be particularly useful to treat cancers in deep tissues in patients that have not responded successfully to conventional treatments. For example, cholangiocarcinoma or bile duct cancers mostly grow along with the biliary ducts and obstruct them, causing liver dysfunction and jaundice that might kill patients [23, 24]. To avoid such complications, patients need to place a biliary stent in those parts of the biliary duct occluded or compressed by the tumor, in order to maintain the biliary flow. In these patients, combined implantation of the wireless NIR-LED along with the biliary stent might at the same time prevent occlusion of the bile duct and, also, treat the cancer in a minimally invasive way. Similarly, wireless powered implantable LED devices might also be combined with external or internal draining tubes and placed temporarily, or implanted following surgery to kill residual cancer cells.

It is clear that further research is needed to fully understand the viability of the proposed device for use in humans as well as to investigate possible issues (e.g., safety, efficacy, compatibility, and optimal criteria for clinical use). Additionally, orthotopic tumor models growing in respective organs are surely superior to the subcutaneously xenografted tumor model used in this study for evaluating clinical relevance of this LED system [25-27]. However, the results here shown are promising as the effectiveness of the proposed implantable LED for NIR-PIT was demonstrated both in in vitro and in vivo.

\section{MATERIALS AND METHODS}

\section{Wireless NIR-LED}

Figure 1 shows the components of the proposed wireless NIR-LED: the circuits of LED capsule and power supply device (Figure 1A); the power supply device (Figure 1B); the LED capsule (Figure 1C); and the two LED in the capsule in light-ON and light-OFF state (Figure 1D). The transmitter coil was made by winding a copper litz wire by six turns. The inner diameter was 150 $\mathrm{mm}$ and the outer diameter was $300 \mathrm{~mm}$. The transmitter coil was connected to a capacitor (resonance frequency 1 $\mathrm{MHz}$ ) and an inverter in series. The inverter applied high-

A

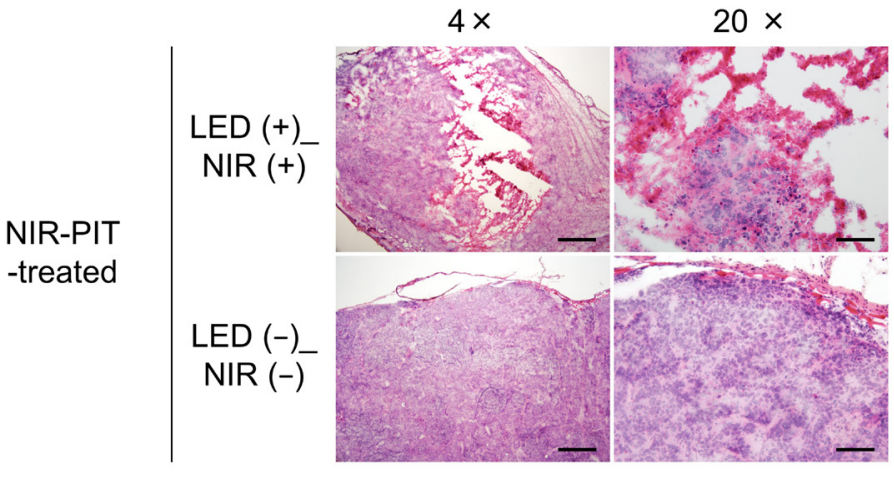

B

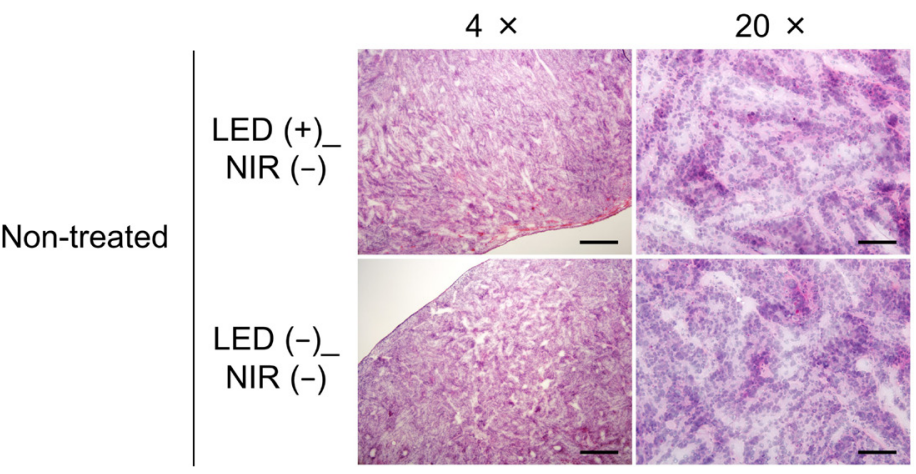

Figure 6: Histological analysis of NIR-PIT effects. (A) Treated mice: tumor cells were significantly damaged in the LED (+)_NIR $(+)$ tumor (top), whereas no apparent damage was observed in LED (-)_NIR (-) tumors (bottom). (B) Non-treated mice: no apparent cell damage was observed, neither in LED (+)_NIR (-) (top) nor in LED (-)_NIR (-) tumors (bottom). Scale bar: $500 \mu \mathrm{m}$ in $4 \times$ images. Scale bar: $100 \mu \mathrm{m}$ in $20 \times$ images. 
frequency current to the coil and capacitor to generate a magnetic field.

The LED capsule was about $7 \mathrm{~mm}$ in length and was composed of a receiver coil, a capacitor and two LEDs as shown in Figure 1A and 1C. The two LEDs (SMT690D; USHIO OPTO SEMICONDUCTORS INC., Kyoto, Japan) emitted light at 680-700 $\mathrm{nm}$. The receiver coil was made by winding a copper wire around a cylindrical ferrite (diameter: $2 \mathrm{~mm}$; length: $5 \mathrm{~mm}$ ) by approximately 30 turns. Coupling of transmitter coil and received coil provided power to the LED with electromagnetic induction. In fact, changes in the magnetic field generated by the transmitter coil induced current flow in the receiver coil thus providing electric power to the LEDs. To limit possible immune responses, the LED capsule was fully coated with biocompatible epoxy resin.

\section{Reagents}

Panitumumab (Vectibix $\left.{ }^{\circledR}\right)$, a fully humanized IgG2 monoclonal antibody (mAb) directed against the epidermal growth factor receptor (EGFR), was purchased from Takeda Pharmaceutical Co. Ltd. (Osaka, Japan). Trastuzumab (Herceptin ${ }^{\circledR}$ ), 95\% humanized IgG1 mAb directed against the human EGFR type-2 (HER2), was purchased from Chugai Pharmaceutical Co., Ltd. (Tokyo, Japan). Water-soluble silicon-phthalocyanine derivative, IRDye700DX NHS ester (IR700) was obtained from Li-COR Bioscience (Lincon, NE, USA). All the other chemicals used were of reagent grade.

\section{Synthesis of IR700 conjugated antibodies}

Panitumumab or Trastuzumab (1 mg, $6.8 \mathrm{nmol})$ was incubated with IR700 (66.8 $\mu \mathrm{g}, 34.2 \mathrm{nmol})$ in $0.1 \mathrm{M} \mathrm{Na}_{2} \mathrm{HPO}_{4}(\mathrm{pH} \mathrm{8.5})$ at room temperature for $2 \mathrm{~h}$. The mixture was purified with a gel-filtration column (Sephadex G50, PD-10; GE Healthcare, Milwaukee, WI, USA), and derived Panitumumab-IR700 (Pan-IR700) or Trastuzumab-IR700 (Tra-IR700). Protein concentration was determined with Modified Lowry Protein Assay Kit (Thermo Fisher Scientific Inc, Rockford, IL, USA) by measuring the absorption at $750 \mathrm{~nm}$ with a microplate reader (Infinite M200; Tecan Austria GmbH, Grödig, Austria). The concentration of IR700 was measured by absorption at $689 \mathrm{~nm}$ with a spectrophotometer (UV-1800; Shimadzu Corp., Kyoto, Japan) to confirm the number of fluorophore molecules to each mAb. The number of IR700 per $\mathrm{mAb}$ was approximately equal to three.

\section{Cell culture}

Green fluorescence protein (GFP), luciferaseexpressing A431 (A431-GFP-luc) cells, and HER2-genetransfected NIH3T3 (3T3-HER2) cells were prepared by Dr. Kobayashi's laboratory. Cell lines were grown in
RPMI-1640 (SIGMA, Saint Louis, MO, USA) containing $10 \%$ fetal bovine serum (Gibco Life Technologies, Grand Island, NY, USA) and 1\% penicillin/streptomycin (Nacalai Tesque Inc., Kyoto, Japan) in tissue culture dishes in a humidified incubator at $37^{\circ} \mathrm{C}$ in an atmosphere composed of air $(95 \%)$ and carbon dioxide (5\%).

\section{In vitro NIR-PIT}

A431-GFP-luc cells or 3T3-HER2 cells were seeded on $35 \mathrm{~mm}$ culture dishes and incubated for $24 \mathrm{~h}$. After washing with phosphate buffered saline (PBS), phenolred-free RPMI-1640 supplemented with 15 mM HEPES was added. Pan-IR700 or Tra-IR700 was then added to the culture medium at $5 \mu \mathrm{g} / \mathrm{mL}$ and incubated for $1 \mathrm{~h}$ at $37^{\circ}$ C. Ethidium homodimer-1 (EthD-1; Molecular Probes, Eugene, OR, USA) was used to detect dead cells. The cells were irradiated from the bottom of the dish with NIR light using the wireless LED for 0, 3, 10, 30, and $60 \min \left(0,0.23,0.78,2.2\right.$, and $\left.4.7 \mathrm{~J} / \mathrm{cm}^{2}\right)$. Power density was measured with an optical power meter (PM121D; Thorlabs, Newton, NJ, USA, $\left.1.3 \mathrm{~mW} / \mathrm{cm}^{2}\right)$. The cells were observed by fluorescence microscope (CKX41; Olympus Corporation, Tokyo, Japan) $2 \mathrm{~h}$ after the onset of light exposure. To detect EthD-1, a combination of 480-550 $\mathrm{nm}$ excitation filter and $590 \mathrm{~nm}$ long pass emission filter was used. To detect IR700, a combination of 673-748 nm excitation filter and $765-855 \mathrm{~nm}$ band pass emission filter was used.

For BLI, after irradiation from the bottom with NIR light using the wireless LED for $1,3,5,10,20$, and 30 $\min \left(0.08,0.23,0.39,0.78,1.5\right.$, and $\left.2.2 \mathrm{~J} / \mathrm{cm}^{2}\right)$, phenolred-free RPMI-1640 containing $150 \mu \mathrm{g} / \mathrm{mL}$ D-luciferin was administered to A431-GFP-luc cells. It was analyzed by using a luminescent imaging analyzer LAS-4000 mini (FujiFilm Life Sciences, Tokyo, Japan). Image analysis was conducted with ImageJ software (http://rsb.info.nih.gov/ij/).

\section{Animal and tumor model}

The experimental protocols were approved by the Hokkaido University Animal Care Committee in accordance with the guidelines for the care and use of laboratory animals. Balb/c Slc-nu/nu nude mice (6-weeks-old, females) were purchased from Japan SLC, Inc. (Shizuoka, Japan). During procedures, mice were anesthetized with isoflurane.

Five million A431-GFP-luc cells in PBS were injected subcutaneously in right and left sides of the dorsum, and the experiments were conducted 6 days following cell injection. The greatest longitudinal diameter (length) and the greatest transversal diameter (width) of tumors were measured by using a caliper. Tumor volume was calculated following [28]: volume $=$ length $\times$ width $^{2} \times 0.5$. Body weight was also measured. IR700 fluorescence was evaluated using the FluorVivo Imaging System (Indec BioSystem, Santa 
Clara, CA, USA) (excitation: 600-640 nm, emission: $665 \mathrm{~nm}$ long pass). The ClearView Imaging System (Indec BioSystem, Santa Clara, CA, USA) was used to obtain fluorescence images of GFP (excitation: 452-487 nm, emission: $500 \mathrm{~nm}$ long pass). The region of interest was manually determined on each tumor area. Fluorescence image analysis was conducted with ImageJ software.

\section{In vivo NIR-PIT}

Mice were given intravenous injection of $100 \mu \mathrm{g}$ of Pan-IR700 on day 0 and day 3. The mice had two tumors on the dorsum and were randomized into two groups: (1) treated mice: a tumor with NIR-LED (LED $(+)$ NIR $(+))$ and a tumor without LED (LED (-) NIR $(-))$; (2) non-treated mice: a tumor with implanted dummy LED (not emitting NIR light) (LED (+)_NIR (-)) and a tumor without LED (LED (-)_NIR (-)) (Figure 4A). The dummy LEDs were used to investigate possible tumor growth inhibition effects due to damage of blood vessels during LED implantation. LED capsules were implanted subcutaneously in a way that the tumor was in between the two LED capsules (Figure 4B). Mice were bred on the wireless power supply device after LED implantation. Figure $4 \mathrm{C}$ shows the treatment and imaging schedule. NIR-LED irradiation of LED $(+)$ NIR $(+)$ tumor in treated mice was continuous until day 6. IR700 fluorescence imaging was assessed on day 1 and day 2 . GFP fluorescence imaging was assessed on day 6 .

\section{Histological analysis}

To evaluate the histological changes, mice were euthanized on day 6. Extracted tumors were frozen in optimal cutting temperature compound, and $10 \mu \mathrm{m}$ frozen slice sections were prepared. Hematoxylin and eosin (H\&E) staining was performed according to standard protocols. The slides were imaged with an Olympus BX41 microscope (Olympus Corporation, Tokyo, Japan) with $4 \times$ and $20 \times$ objective lenses.

\section{Statistical analysis}

The measured quantities were expressed as means \pm SEM (standard error of the mean). Statistical analyses were carried out using JMP Pro 12.2.0 software (SAS Institute Inc., Cary, NC, USA). Possible differences in mean values were assessed by using student's $t$ tests. Values of $p<0.05$ were considered statistically significant.

\section{Abbreviations}

BLI: bioluminescence imaging; EGFR: epidermal growth factor receptor; EthD-1: ethidium homodimer-1; HER2: human epidermal growth factor receptor type2; ICNIRP: International Commission on Non-Ionizing Radiation Protection; IR700: IRDye700DX; LED: light emitting diode; mAb: monoclonal antibody; NIR-PIT: near-infrared photoimmunotherapy.

\section{Author contributions}

K.N. conducted experiments, performed the analysis, and wrote the manuscript. T.K. planed and initiated the project, developed the wireless LED system, and wrote the manuscript. H.T. and K.S conducted in vitro experiments and performed the analysis. Y.Y., A.K., and T.S. developed the wireless LED system. H.K. and M.O. planed and initiated the project, designed experiments, wrote the manuscript, and supervised the project.

\section{ACKNOWLEDGMENTS}

The authors thank Prof. Makoto Mitsunaga, Jikei Medical University for valuable comments on the clinical applications.

\section{CONFLICTS OF INTEREST}

The authors declare no conflicts of interest.

\section{FUNDING}

This research was supported by PRESTO, Japan Science and Technology Agency (JPMJPR15P5). This work was also supported by the Intramural Research Program of the National Institutes of Health, National Cancer Institute, Center for Cancer Research.

\section{REFERENCES}

1. Mitsunaga M, Ogawa M, Kosaka N, Rosenblum LT, Choyke PL, Kobayashi H. Cancer cell-selective in vivo near infrared photoimmunotherapy targeting specific membrane molecules. Nat Med. 2011; 17:1685-1691.

2. Sato K, Nagaya T, Nakamura Y, Harada T, Choyke PL, Kobayashi H. Near infrared photoimmunotherapy prevents lung cancer metastases in a murine model. Oncotarget. 2015; 6:19747-19758. https://doi.org/10.18632/oncotarget.3850.

3. Kobayashi H, Ogawa M, Alford R, Choyke PL, Urano Y. New Strategies for Fluorescent Probe Design in Medical Diagnostic Imaging. Chem Rev. 2010; 110:2620-2640.

4. Plaetzer K, Krammer B, Berlanda J, Berr F, Kiesslich T. Photophysics and photochemistry of photodynamic therapy: Fundamental aspects. Lasers Med Sci. 2009; 24:259-268.

5. Sato K, Watanabe R, Hanaoka H, Nakajima T, Choyke PL, Kobayashi H. Comparative effectiveness of light emitting diodes (LEDs) and Lasers in near infrared photoimmunotherapy. Oncotarget. 2016; 7:14324-14335. https://doi.org/10.18632/oncotarget.7365.

6. Okuyama S, Nagaya T, Sato K, Ogata F, Maruoka Y, Choyke PL, Kobayashi H. Interstitial near-infrared 
photoimmunotherapy: effective treatment areas and light doses needed for use with fiber optic diffusers. Oncotarget. 2018; 9:11159-11169. https://doi.org/10.18632/ oncotarget. 24329 .

7. Mitsunaga M, Nakajima T, Sano K, Choyke PL, Kobayashi H. Near-infrared theranostic photoimmunotherapy (PIT): Repeated exposure of light enhances the effect of immunoconjugate. Bioconjug Chem. 2012; 23:604-609.

8. Sato K, Nagaya T, Mitsunaga M, Choyke PL, Kobayashi H. Near infrared photoimmunotherapy for lung metastases. Cancer Lett. 2015; 365:112-21.

9. Sullivan CR. Optimal choice for number of strands in a litz-wire transformer winding. IEEE Transactions on Power Electronics. 1999; 14:283-291.

10. Sato M, Yamamoto G, Gunji D, Imura T, Fujimoto H. Development of Wireless In-Wheel Motor Using Magnetic Resonance Coupling. IEEE Transactions on Power Electronics. 2016; 31:5270-5278.

11. Mitsunaga M, Nakajima T, Sano K, Kramer-Marek G, Choyke PL, Kobayashi H. Immediate in vivo target-specific cancer cell death after near infrared photoimmunotherapy. BMC Cancer. 2012; 12:345.

12. Sato K, Nagaya T, Choyke PL, Kobayashi H. Near infrared photoimmunotherapy in the treatment of pleural disseminated NSCLC: Preclinical experience. Theranostics. 2015; 5:698-709.

13. Hoffman RM. The multiple uses of fluorescent proteins to visualize cancer in vivo. Nat Rev Cancer. 2005; 5:796-806.

14. Sato K, Choyke PL, Kobayashi H. Photoimmunotherapy of gastric cancer peritoneal carcinomatosis in a mouse model. PLoS One. 2014; 9.

15. International Commission on Non-Ionizing Radiation Protection. Guidelines for limiting exposure to time-varying electric and magnetic fields ( $1 \mathrm{~Hz}$ to $100 \mathrm{kHz}$ ). Health Phys. 2010; 99:818-36. Erratum in: Health Phys. 2011; 100:112.

16. Kim JD, Sun C, Suh IS. A proposal on wireless power transfer for medical implantable applications based on reviews. 2014 IEEE Wireless Power Transfer Conference. 2014; 166-9.

17. Joung YH. Development of Implantable Medical Devices: From an Engineering Perspective. Int Neurourol J. 2013; 17:98.

18. Nakajima T, Sato K, Hanaoka H, Watanabe R, Harada T, Choyke PL, Kobayashi H. The effects of conjugate and light dose on photo-immunotherapy induced cytotoxicity. BMC Cancer. 2014; 14:389.

19. Maruoka Y, Nagaya T, Nakamura Y, Sato K, Ogata F, Okuyama S, Choyke PL, Kobayashi H. Evaluation of early therapeutic effects after near infrared photoimmunotherapy (NIR-PIT) using luciferase-luciferin photon-counting and fluorescence imaging. Mol Pharm. 2017; 14:4628-4635.

20. Sano K, Nakajima T, Choyke PL, Kobayashi H. The effect of photoimmunotherapy followed by liposomal daunorubicin in a mixed tumor model: a demonstration of the super-enhanced permeability and retention effect after photoimmunotherapy. Mol Cancer Ther. 2014; 13:426-432.

21. Nagaya T, Nakamura Y, Sato K, Harada T, Choyke PL, Kobayashi H. Improved micro-distribution of antibodyphoton absorber conjugates after initial near infrared photoimmunotherapy (NIR-PIT). J Control Release. 2016; 232:1-8.

22. Sano K, Nakajima T, Choyke PL, Kobayashi H. Markedly Enhanced Permeability and Retention E ff ects Induced by Photo-immunotherapy of Tumors. ACS Nano. 2013; 7:717-724.

23. Seyama Y, Kubota K, Sano K, Noie T, Takayama T, Kosuge T, Makuuchi M. Long-Term Outcome of Extended Hemihepatectomy for Hilar Bile Duct Cancer with No Mortality and High Survival Rate. Ann Surg. 2003; 238:73-83.

24. Tompkins RK, Thomas D, Wile A, Longmire WP. Prognostic factors in bile duct carcinoma: analysis of 96 cases. Ann Surg. 1981; 194:447-457.

25. Hoffman RM. Orthotopic metastatic mouse models for anticancer drug discovery and evaluation: A bridge to the clinic. Invest New Drugs. 1999; 17:343-359.

26. Hoffman RM. Patient-derived orthotopic xenografts: Better mimic of metastasis than subcutaneous xenografts. Nat Rev Cancer. 2015; 15:451-452.

27. Hoffman RM. Orthotopic Is Orthodox: Why Are Orthotopic-Transplant Metastatic Models Different From All Other Models? J Cell Biochem. 1994; 56:1-3.

28. Euhus DM, Hudd C, LaRegina MC, Johnson FE. Tumor measurement in the nude mouse. J Surg Oncol. 1996; 31:229-234. 